\title{
GammaCHI: a package for the inversion and computation of the gamma and chi-square cumulative distribution functions (central and noncentral)
}

\author{
Amparo Gil $^{\mathrm{a}}$, Javier Segura ${ }^{\mathrm{b}}$, Nico M. Temme ${ }^{\mathrm{c}}$ \\ ${ }^{a}$ Depto. de Matemática Aplicada y Ciencias de la Comput. Universidad de Cantabria. \\ 39005-Santander, Spain.e-mail: amparo.gil@unican.es \\ ${ }^{b}$ Depto. de Matemáticas, Estadística y Comput. Universidad de Cantabria. \\ 39005-Santander, Spain \\ ${ }^{c}$ IAA, 1391 VD 18, Abcoude, The Netherlands ${ }^{1}$
}

\begin{abstract}
A Fortran 90 module GammaCHI for computing and inverting the gamma and chi-square cumulative distribution functions (central and noncentral) is presented. The main novelty of this package are the reliable and accurate inversion routines for the noncentral cumulative distribution functions. Additionally, the package also provides routines for computing the gamma function, the error function and other functions related to the gamma function. The module includes the routines cdfgamC, invcdfgamC, cdfgamNC, invcdfgamNC, errorfunction, inverfc, gamma, loggam, gamstar and quotgamm for the computation of the central gamma distribution function (and its complementary function), the inversion of the central gamma distribution function, the computation of the noncentral gamma distribution function (and its complementary function), the inversion of the noncentral gamma distribution function, the computation of the error function and its complementary function, the inversion of the complementary error function, the computation of: the gamma function, the logarithm of the gamma function, the regulated gamma function and the ratio of two gamma functions, respectively.
\end{abstract}

\section{PROGRAM SUMMARY}

\footnotetext{
${ }^{1}$ Former address: CWI, 1098 XG Amsterdam, The Netherlands
} 
Manuscript Title:

GammaCHI: a package for the inversion and computation of the gamma and chisquare cumulative distribution functions (central and noncentral)

Authors:

Amparo Gil, Javier Segura, Nico M. Temme

Program Title:

Module GammaCHI

Journal Reference:

Catalogue identifier:

Licensing provisions:

Programming language:

Fortran 90

Computer:

Any supporting a FORTRAN compiler.

Operating system:

Any supporting a FORTRAN compiler.

$R A M$ :

a few MB

Number of processors used:

Keywords:

Gamma cumulative distribution function; chi-square cumulative distribution function; inversion of cumulative distribution functions; error function; complementary error function; gamma function; logarithm of the gamma function; regulated gamma function; quotient of gamma functions.

Classification:

4.7 Other functions.

External routines/libraries:

None.

Subprograms used:

Nature of problem:

The computation and inversion of gamma and chi-square cumulative distribution functions (central and noncentral) as well as the computation of the error and gamma functions is needed in many problems of applied and mathematical physics.

Solution method:

The algorithms use different methods of computation depending on the range of parameters: asymptotic expansions, quadrature methods, etc.

Restrictions:

In the inversion of the central gamma/chi-square distribution functions, very 
small input function values $P_{\mu}(x, y), Q_{\mu}(x, y)$ (lower than $10^{-150}$ ) are not admissible.

The admissible input parameter ranges for computing the noncentral cumulative gamma distribution functions $P_{\mu}(x, y), Q_{\mu}(x, y)$ in standard IEEE double precision arithmetic are $0 \leq x \leq 10000,0 \leq y \leq 10000,0.5 \leq \mu \leq 10000$ and the related parameter ranges for the noncentral chi-square cumulative distribution function. In the inversion of the noncentral gamma/chi-square distribution functions, very small input function values $P_{\mu}(x, y), Q_{\mu}(x, y)$ (lower than $10^{-25}$ and $10^{-35}$, respectively) are not admissible.

Running time:

It varies depending on the function and the parameter range.

\section{Introduction}

The Fortran 90 module GammaCHI provides reliable and fast routines for the inversion and computation of the gamma and chi-square distribution functions. These functions appear in many problems of applied probability including, of course, a large number of problems in Physics. The module also includes routines for the computation of the gamma function, the error function and its complementary function, the inverse of the complementary error function, the logarithm of the gamma function, the regulated gamma function and the ratio of two gamma functions.

The main novelty of the package GammaCHI are the inversion routines for the noncentral cumulative distribution functions. These algorithms are based on the asymptotic methods presented in [1] although improvements in the estimation of the initial values for small values of the parameter $\mu$ are included in the present algorithms. Among other applications, the computation and the inversion of the noncentral cumulative distribution functions included in GammaCHI appear in the analysis of signal detection in different physical scenarios such as optics [2], radiometry [3] or quantum detection [4], [5]. Also, the inversion routines can be used to generate normal variates as well as central and noncentral gamma (or chi-square) variates, which can be useful, for example, in Monte Carlo simulations.

\section{Theoretical background}

\subsection{Central gamma distribution function}

The incomplete gamma functions are defined by 


$$
\gamma(a, x)=\int_{0}^{x} t^{a-1} e^{-t} d t, \quad \Gamma(a, x)=\int_{x}^{\infty} t^{a-1} e^{-t} d t .
$$

Let us define

$$
P(a, x)=\frac{1}{\Gamma(a)} \gamma(a, x), \quad Q(a, x)=\frac{1}{\Gamma(a)} \Gamma(a, x),
$$

where we assume that $a$ and $x$ are real positive numbers.

The functions $P(a, x), Q(a, x)$ are the central gamma distribution function and its complementary function, respectively. These distribution functions which appear in many problems of applied probability include, as particular cases, the standard chi-square probability functions $P\left(\chi^{2} \mid \nu\right)$ and $Q\left(\chi^{2} \mid \nu\right)$ with parameters $a=\nu / 2$ and $x=\chi^{2} / 2$.

The central gamma distribution functions satisfy the complementary relation

$$
P(a, x)+Q(a, x)=1 .
$$

In hypothesis testing, and using the chi-square distribution, it is usual to consider the problem as $Q\left(\chi^{2} \mid \nu\right)=\alpha ; \alpha$ is the probability that a variable distributed according to the chi-square distribution with $\nu$ degrees of freedom exceeds the value $\chi^{2}$. Two kinds of problems are usually considered: a) computing the confidence level $\alpha$ given an experimentally determined chisquare value $\chi^{2}$; b) computing percentage points for certain values of $\alpha$ and for different degrees of freedom $\nu$ (the standard tables which are common in statistics text-books usually include this information). The first problem involves the direct computation of the cumulative distribution function while the second one is an inversion problem.

The module GammaCHI includes routines both for the direct computation of the distribution functions (central gamma/chi-square) and their inversion. Regarding the direct computation, the routine first computes $\min \{P(a, x), Q(a, x)\}$, and the other one by using (3) . Computing the $Q(a, x)$ function simply as $1-P(a, x)$ when $P(a, x)$ is close to 1 can lead to serious cancellation problems, which are avoided in our algorithms.

On the other hand, the inversion routine solves the equations

$$
P(a, x)=p, \quad Q(a, x)=q, \quad 0<p, q<1,
$$

for a given value of $a$. 
The algorithm for computing the gamma distribution function and its inversion is described in [6]. The computation of the distribution functions is based on the use of Taylor expansions, continued fractions or uniform asymptotic expansions, depending on the parameter values. For the inversion, asymptotic expansions in combination with high-order Newton methods are used.

The version included in the package GammaCHI for the inversion includes some improvements in its performance for small values of $p$ and $q$. For such small values, some of the elementary bounds for incomplete gamma functions given in [7] provide accurate enough approximations for estimating sufficiently accurate starting values for the Newton iterative process.

\subsection{Noncentral gamma distribution function}

The noncentral gamma cumulative distribution functions can be defined as

$$
\begin{aligned}
& P_{\mu}(x, y)=e^{-x} \sum_{k=0}^{\infty} \frac{x^{k}}{k !} P(\mu+k, y), \\
& Q_{\mu}(x, y)=e^{-x} \sum_{k=0}^{\infty} \frac{x^{k}}{k !} Q(\mu+k, y),
\end{aligned}
$$

where $P(\mu, y)$ and $Q(\mu, y)$ are the central gamma cumulative distribution function and its complementary function, respectively; $x$ is called the noncentrality parameter of the distribution functions.

As in the case of the central distribution functions, the noncentral functions satisfy the relation

$$
P_{\mu}(x, y)+Q_{\mu}(x, y)=1 .
$$

On the other hand, if $\chi_{n}^{2}(\lambda)$ is a random variable with a noncentral chisquare distribution with $n>0$ degrees of freedom and noncentrality parameter $\lambda \geq 0$, then the lower and upper tail probabilities for the distribution function of $\chi_{n}^{2}(\lambda)$ are given by

$$
\begin{aligned}
& \operatorname{Prob}\left(\chi_{n}^{2}(\lambda)<t\right)=e^{-\lambda / 2} \sum_{k=0}^{\infty} \frac{(\lambda / 2)^{k}}{k !} P(n / 2+k, t / 2), \\
& \operatorname{Prob}\left(\chi_{n}^{2}(\lambda)>t\right)=e^{-\lambda / 2} \sum_{k=0}^{\infty} \frac{(\lambda / 2)^{k}}{k !} Q(n / 2+k, t / 2),
\end{aligned}
$$


from which, and comparing with (5), it follows the simple relation between distribution functions: if the random variable $Y$ has a noncentral gamma distribution with parameters $a$ and noncentrality parameter $\lambda$, then $X=2 Y$ has a noncentral chi-square distribution with parameter $n=2 a$ and with noncentrality parameter $2 \lambda$.

Algorithms for computing the functions $P_{\mu}(x, y)$ and $Q_{\mu}(x, y)$ for a large range of the parameters $\mu(\mu \geq 1), x, y$ are described in [8]. The methods include series expansions in terms of the incomplete gamma functions, recurrence relations, continued fractions, asymptotic expansions, and numerical quadrature. In the module GammaCHI the lower $\mu$ range of computation of the algorithm for computing the noncentral gamma cumulative distribution function is extended to include the interval $\frac{1}{2} \leq \mu<1$. For extending the algorithms to this interval, we use a single step of the following three-term homogeneous recurrence relation (Eq.14 of [8])

$$
y_{\mu+1}-\left(1+c_{\mu}\right) y_{\mu}+c_{\mu} y_{\mu-1}=0, \quad c_{\mu}=\sqrt{\frac{y}{x}} \frac{I_{\mu}(2 \sqrt{x y})}{I_{\mu-1}(2 \sqrt{x y})} .
$$

Both $Q_{\mu}(x, y)$ and $P_{\mu}(x, y)$ satisfy (8). In this expression, ratios of Bessel functions appear. These ratios are efficiently computed using a continued fraction representation.

Regarding the inversion of the noncentral gamma/chi-square distribution functions, two different kinds of inversion problems can be considered:

Problem 1: Find $x$ from the equation

$$
Q_{\mu}(x, y)=q
$$

with fixed $y, \mu$ and $q$.

Problem 2: Find $y$ from the equation

$$
P_{\mu}(x, y)=p
$$

with fixed $x, \mu$ and $p$.

The inversion of $Q_{\mu}(x, y)$ with respect to $x$ corresponds to the problem of inverting the distribution function with respect to the noncentrality parameter given the upper tail probability. On the other hand, the inversion of $P_{\mu}(x, y)$ with respect to $y$ with fixed $x$ corresponds to the problem of 
computing the $p$-quantiles of the distribution function. For noncentral chisquare distributions, the inversion with respect to $y$ allows the computation of a variate with a given number of degrees of freedom and a given noncentrality parameter.

In the inversion with respect to $x$, it is important to note that not for all the possible values of the pair $\left(y_{0}, q_{1}\right)$ there is a solution of the equation

$$
Q_{\mu}\left(x, y_{0}\right)=q_{1} \text {. }
$$

In particular, the values of $q_{1}$ such that $q_{1}<q_{0}$, where $q_{0}=Q_{\mu}\left(0, y_{0}\right)=$ $Q_{\mu}\left(y_{0}\right)$, do not make sense because $Q_{\mu}(x, y)$ is increasing as a function of $x$.

Both in the inversion with respect to $x$ and $y$, a combination of asymptotic expansions and secant methods can be used, as discussed in [1]. A Fortran 90 implementation is now available in the module GammaCHI. As for the direct computation of function distribution values, in this implementation the range of computation for the inversion of the gamma cumulative distribution function is extended to include the interval $\frac{1}{2} \leq \mu<1$. The computation in this interval is made by using the double asymptotic property of the expansions derived in [1]: the formulas obtained in [1] can all be used for small values of $\mu$ if $\xi=2 \sqrt{x y}$ is large. More details can be found in the appendix. For small $q$ this is suplemented with estimations for the case $\mu=1 / 2$, which can be written in terms of error functions; we use the result of the inversion for the case $\mu=1 / 2$ to estimate a starting value when $\mu$ is close to $1 / 2$. It is easy to check that

$$
Q_{1 / 2}(x, y)=\frac{1}{2}(\operatorname{erfc}(\sqrt{x}+\sqrt{y})+\operatorname{erfc}(\sqrt{y}-\sqrt{x}))
$$

and for this function, computing derivatives is simple and one can apply the fourth order method described in [9].

\subsection{Error function and its complementary function}

The error function $\operatorname{erf}(x)$ [10] is defined by the integral

$$
\operatorname{erf}(x)=\frac{2}{\sqrt{\pi}} \int_{0}^{x} e^{-t^{2}} d t
$$

Its complement with respect to 1 is denoted by

$$
\operatorname{erfc}(x)=1-\operatorname{erf}(x)=\frac{2}{\sqrt{\pi}} \int_{x}^{\infty} e^{-t^{2}} d t
$$


The complementary error function is very closely related to the normal distribution functions, which are defined by

$$
P(x)=\frac{1}{\sqrt{2 \pi}} \int_{-\infty}^{x} e^{-t^{2} / 2} d t, Q(x)=\frac{1}{\sqrt{2 \pi}} \int_{x}^{\infty} e^{-t^{2} / 2} d t
$$

with the property $P(x)+Q(x)=1$.

From the definition of the complementary error function it follows that

$$
P(x)=\frac{1}{2} \operatorname{erfc}(-x / \sqrt{2}), Q(x)=\frac{1}{2} \operatorname{erfc}(x / \sqrt{2}) .
$$

The algorithm for the error functions is based on the use of rational approximations [11] and it includes the possibility of computing the scaled complementary error function $e^{x^{2}} \operatorname{erfc}(x)$. This is important to avoid underflow problems in numerical computations of the complementary error function (the dominant exponential behavior of the function is $e^{-x^{2}}$ ). In general, it is quite useful (when possible) to define scaled values of mathematical functions with the dominant exponential behavior factored out [12, §12.1.3].

Our module includes also the computation of the inverse of the complementary error function $y=\operatorname{inverfc} x$. Using the relation (15), this routine can be also useful in the generation of normal variates.

For values of $x$ close to 1 , the following expansion is used

$$
\text { inverfc } x=t+\frac{1}{3} t^{3}+\frac{7}{30} t^{5}+\frac{127}{630} t^{7}+\ldots, \quad 0<x<2 .
$$

with $t=\frac{1}{2} \sqrt{\pi}(1-x)$. For these and more coefficients, see [13].

\subsection{Gamma function}

Three standard definitions of the gamma function are usually considered:

$$
\begin{gathered}
\Gamma(z)=\int_{0}^{\infty} e^{-t} t^{z-1} d t, \operatorname{Re} z>0 \text { (Euler). } \\
\Gamma(z)=\lim _{n \rightarrow \infty} \frac{n ! n^{z}}{z(z+1) \cdots(z+n)}, z \neq 0,-1,-2, \ldots \text { (Euler). } \\
\frac{1}{\Gamma(z)}=z e^{\gamma z} \prod_{n=1}^{\infty}\left(1+\frac{z}{n}\right) e^{-z / n} \text { (Weierstrass), }
\end{gathered}
$$

with $\gamma=0.57721 \ldots$, Euler's constant. The equivalence of these definitions is proved in [14]. 
Important relations are

$$
\Gamma(z+1)=z \Gamma(z), \quad(\text { recursion })
$$

and

$$
\frac{1}{\Gamma(1+z) \Gamma(1-z)}=\frac{\sin \pi z}{\pi z}
$$

which is called the reflection formula. This formula is important for range reduction and it is easily proved by using (19).

For computing the gamma function, we use recursion for small values of $x$ and the relation given in (22) for larger values. The special cases $x=n$ and $x=n+\frac{1}{2}$ are treated separately.

\subsubsection{Logarithm of the gamma function}

The following representation of $\log \Gamma(z)$ is very important for deriving expansions for large values of $|z|$ :

$$
\log \Gamma(z)=\left(z-\frac{1}{2}\right) \log z-z+\frac{1}{2} \log (2 \pi)+S(z) .
$$

For large $|z|, S(z)$ gives a small correction with respect to the remaining terms of the right-hand side. Neglecting $S(z)$ gives the well-known Stirling formula

$$
\Gamma(z) \sim z^{z} e^{-z}\left(\frac{2 \pi}{z}\right)^{1 / 2}, z \rightarrow \infty .
$$

$S(z)$ can be written in terms of an expansion involving Bernoulli numbers:

$$
S(z)=\sum_{n=0}^{N-1} \frac{B_{2 n+2}}{(2 n+1)(2 n+2) z^{2 n+1}}+E_{N}(z) .
$$

The Bernoulli numbers $B_{n}$ are given by $B_{n}=B_{n}(0)$. The first few are $B_{0}=1, B_{1}=-\frac{1}{2}, B_{2}=\frac{1}{6}, B_{3}=0, B_{4}=-\frac{1}{30}$. More information on Bernoulli polynomials and Bernoulli numbers can be found in [15, §24.2(i)].

A bound for $\left|E_{N}\right|$ in (24) can be obtained as

$$
\left|E_{N}\right| \leq \frac{B_{2 N+2} K(z)}{(2 N+1)(2 N+2)}|z|^{-2 N-1},
$$


where

$$
K(z)=\sup _{u \geq 0}\left|\frac{z^{2}}{z^{2}+u^{2}}\right| .
$$

If $|\arg z|<\pi / 4$, then $K(z)=1$. For real positive $z, E_{N}(z)$ is less in absolute value than the first term neglected in (24) and it has the same sign. From (25) it follows that $E_{N}(z)=\mathcal{O}\left(z^{-2 N-1}\right)$ for Re $z \rightarrow+\infty$. Inserting the values of the first Bernoulli numbers we arrive at the representation (Stirling's series):

$$
\begin{aligned}
\log \Gamma(z)= & \left(z-\frac{1}{2}\right) \log z-z+\frac{1}{2} \log (2 \pi)+ \\
& \frac{1}{12 z}-\frac{1}{360 z^{3}}+\frac{1}{1260 z^{5}}-\frac{1}{1680 z^{7}}+\mathcal{O}\left(z^{-9}\right) .
\end{aligned}
$$

The algorithm for the logarithm of the gamma function makes use of the representation (22). The computation of $S(z)$ is based on the use of the sum (22), Chebyshev series and a minimax rational approximation. Coefficients of rational approximations are taken from the the tables in [16, §6.6]. On some intervals we use Chebyshev expansions, with coefficients derived by using high precision Maple codes.

\subsection{The function $\Gamma^{*}(x)$}

This function (the regulated gamma function) is defined by

$$
\Gamma^{*}(x)=\frac{\Gamma(x)}{\sqrt{2 \pi / x} x^{x} e^{-x}}, \quad x>0 .
$$

When $x$ is large, this function can be very important in algorithms where the function $\Gamma(x)$ is involved because $\Gamma^{*}(x)=1+\mathcal{O}(1 / x)$, as can be seen from its asymptotic expansion (Stirling series):

$$
\Gamma^{*}(x) \sim 1+\frac{1}{12} x^{-1}+\frac{1}{288} x^{-2}+\ldots, \quad x \rightarrow \infty .
$$

The algorithm for computing $\Gamma^{*}(x)$, uses the relation $\Gamma^{*}(x)=\exp (S(x))$ for $x>3$, where $S(x)$ is given in (24). The computation of $S(x)$ was described in the previous section. 


\subsection{The quotient of two gamma functions}

The quotient of two gamma functions appears frequently in applications. From a computational point of view, problems can arise when trying to compute directly the ratio of functions in the case when the arguments of both functions are large. For that reason, a key point in our algorithm for computing the quotient of two gamma functions is the use of the following asymptotic expansion [17, p. 68]:

$$
\frac{\Gamma(x+a)}{\Gamma(x+b)} \sim w^{a-b} \sum_{n=0}^{\infty}(-1)^{n} C_{n} \frac{\Gamma(b-a+2 n)}{\Gamma(b-a)} \frac{1}{w^{2 n}}, \text { as } x \rightarrow \infty,
$$

with $w=x+(a+b-1) / 2$ and $b>a$.

The first five coefficients $C_{n}$ appearing in (29) are:

$$
\begin{aligned}
& C_{0}=1, \\
& C_{1}=\frac{\rho}{12}, \\
& C_{2}=\frac{\rho}{1440}+\frac{\rho^{2}}{288}, \\
& C_{3}=\frac{\rho}{90720}+\frac{\rho^{2}}{17280}+\frac{\rho^{3}}{10368}, \\
& C_{4}=\frac{\rho}{4838400}+101 \frac{\rho^{2}}{87091200}+\frac{\rho^{3}}{414720}+\frac{\rho^{4}}{497664},
\end{aligned}
$$

with $\rho=(a-b+1) / 2$.

\section{Overview of the software structure}

The Fortran 90 package includes the main module GammaCHI, which includes as public routines the following functions and routines: cdfgamC, invcdfgamC, cdfgamNC, invcdfgamNC, errorfunction, inverfc, gamma, loggam, gamstar and quotgamm.

In the module GammaCHI, the auxiliary module Someconstants is used. This is a module for the computation of the main constants used in the different routines.

\section{Description of the individual software components}

1. cdfgamC:

The calling sequence of this routine is 


$$
\text { cdfgamC(ichi, a, x, p, q,ierr) }
$$

where the input data are: $i c h i$ (flag), $a$ and $x$ (arguments of the distribution function). $i c h i$ is a flag for the choice of a gamma or chi-square cumulative distribution function: when $i c h i=1$, the arguments are of a gamma distribution function; when $i c h i=2$, the arguments correspond to a central chi-square distribution function.

The outputs of the function are error flag ierr, the function distribution value $p$ and its complementary function $q$. The possible values of the error flag are: ierr $=0$, successful computation; ierr $=1$, computation failed due to overflow/underflow; ierr $=2$, arguments out of range.

2. invcdfgamC:

The calling sequence of this routine is

$$
\text { invcdfgamC (ichi, a, p, q, xr, ierr) }
$$

where the input data are: $i c h i$ (flag), $a, p$ and $q$. $i c h i$ is a flag for the choice of a gamma or chi-square cumulative distribution function: when $i c h i=1$, a gamma distribution function is inverted; when $i c h i=2$, a central chi-square distribution function is inverted.

The outputs of the function are $x r$ (the solution of the equations $P(a, x r)=p$ and $Q(a, x r)=q)$ and the error flag ierr. The possible values of the error flag are: ierr $=0$, computation successful; $i e r r=1$, overflow problems in one or more steps of the computation; ierr $=2$, the number of iterations in the Newton method reached the upper limit $N=35$; ierr $=3$, any of the arguments of the function is out of range.

3. cdfgamNC:

The calling sequence of this routine is

cdfgamNC(ichi, mu, x, y, p, q, ierr)

where the input data are: $i c h i$ (flag), $m u, x$ and $y$ (arguments of the distribution function). The input $i c h i$ input is a flag for the choice of a gamma or chi-square noncentral cumulative distribution function: when $i c h i=1$, the arguments are of a gamma distribution function; when $i c h i=2$, the arguments correspond to a noncentral chi-square distribution function. 
The outputs of the function are: the function distribution value $p$ and its complementary function $q$ and an error flag ierr. The possible values of the error flag are: ierr $=0$, successful computation; ierr $=1$, computation failed due to overflow/underflow; ierr $=2$, arguments out of range.

\section{4. invcdfgamNC:}

The calling sequence of this routine is

invcdfgamNC(ichi, icho, mu, p, q, yx, xy, ierr)

where the input data are: $i c h i, i c h o, m u, p, q$ and $y x$. $i c h i$ is a flag for the choice of a noncentral gamma or chi-square cumulative distribution function: when $i c h i=1$, the arguments are of a gamma distribution function; when $i c h i=2$, the arguments correspond to a central chisquare distribution function. The input icho is a flag for the choice of the inversion process: if $i c h o=1, x$ is computed in the equations $Q_{m u}(x, y x)=q, P_{m u}(x, y x)=p$. If $i c h o=2, y$ is computed in the equations $Q_{m u}(y x, y)=q, P_{m u}(y x, y)=p ; q$ and $p$ are the values of the cumulative distribution functions; $m u$ and $y x$ are arguments of the distribution functions.

The outputs of the routine are the value obtained in the inversion $x y$ and an error flag ierr. The possible values of the error flags are: ierr $=0$, computation successful; ierr $=1$, in the inversion process with respect to the non-centrality parameter ( $x$-variable), the input values $(y, q)$ do not make sense (i.e. the inequality $q<Q_{m u}(0, y)$ holds); $i e r r=2$, at least one of the gamma distribution function values needed in the inversion process cannot be correctly computed; ierr $=3$, the number of iterations in the secant method reached the upper limit; $i e r r=4$, any of the input arguments is out of range.

\section{5. errorfunction:}

The calling sequence of this function is

errorfunction ( $x, \operatorname{erfc}$, expo)

where the input data are: $x$ (argument of the function), erfc and expo (logical variables).

The logical variables erfc and expo mean the following: 
When erfc=.true. and expo=.false., the function computes the complementary error function $\operatorname{erfc}(x)$.

When erfc $=$.true., expo=.true. and $x>0$, the function computes the scaled complementary error function $e^{x^{2}} \operatorname{erfc}(x)$.

When erfc $=$.false. and expo=false., the function computes the error function $\operatorname{erf}(x)$.

6. inverfc:

The calling sequence of this function is inverfc $(x)$

The input argument is $x$, a positive real number. This function computes the inverse of the complementary error function.

7. gamma:

The calling sequence of this function is

$\operatorname{gamma}(\mathrm{x})$

This function computes the Euler gamma function $\Gamma(x)$, for $x$ real.

8. loggam:

The calling sequence of this function is

$$
\operatorname{loggam}(\mathrm{x})
$$

This function computes $\log (\Gamma(x))$, for $x>0$.

9. gamstar:

The calling sequence of this function is

$\operatorname{gamstar}(\mathrm{x})$

This function computes for positve $x$ the regulated gamma function $\Gamma^{*}(x)=\frac{\Gamma(x)}{\sqrt{2 \pi / x} x^{x} e^{-x}}$.

\section{0. quotgamm:}

The calling sequence of this function is

$$
\text { quotgamm }(x, y)
$$

This function computes $\frac{\Gamma(x)}{\Gamma(y)}$ for $x, y$ real values. 


\begin{tabular}{cccc}
$a$ & $x$ & $Q(a, x)$ & gammainc $\left(x, a,^{\prime}\right.$ upper $\left.^{\prime}\right)$ \\
\hline $10^{-250}$ & $6.310^{-15}$ & $\mathbf{3 . 2 1 2 1 0 1 1 0 9 6 6 1 1 6 7 1 0 ^ { - 2 4 9 }}$ & $3.21210110966116710^{-249}$ \\
$10^{-250}$ & $7.110^{-7}$ & $\mathbf{1 . 3 5 8 0 7 8 5 9 1 2 0 0 9 3 9 3 1 0 ^ { - 2 4 9 }}$ & $1.3580785912009310^{-249}$ \\
$10^{-250}$ & 0.01 & $\mathbf{4 . 0 3 7 9 2 9 5 7 6 5 3 8 1 1 3 5 1 0 ^ { - 2 5 0 }}$ & $4.03792957653811410^{-250}$ \\
$10^{-14}$ & $6.310^{-15}$ & $\mathbf{3 . 2 1 2 1 0 1 1 0 9 6 6 0 6 5 1 1 0 ^ { - 1 3 }}$ & $3.21210110966065210^{-13}$ \\
$10^{-14}$ & $7.110^{-7}$ & $\mathbf{1 . 3 5 8 0 7 8 5 9 1 2 0 0 8 4 8} 10^{-13}$ & $1.35807859120084810^{-13}$ \\
$10^{-14}$ & 0.01 & $\mathbf{4 . 0 3 7 9 2 9 5 7 6 5 3 8 0 4 0 5 1 0 ^ { - 1 4 }}$ & $4.03792957653803910^{-14}$ \\
\hline
\end{tabular}

Table 1. Test of the routine cdfgamC for small values of $a$ and $x$ : comparison of the values obtained for $Q(a, x)$ using our routine cdfgamC and the Matlab function gammainc included in the 2013a release. It should be noted that our value for $x=0.01$, $a=10^{-14}$ agrees in 16 digits with the value obtained with the Mathematica function

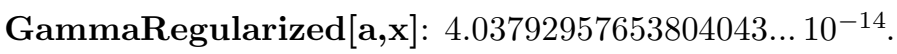

\section{Testing the algorithms}

For testing the algorithm for the computation of the central gamma distribution functions, we use the recurrence relations (see [18, §8.8])

$$
P(a+1, x)=P(a, x)-D(a, x), \quad Q(a+1, x)=Q(a, x)+D(a, x),
$$

where

$$
D(a, x)=\frac{x^{a} e^{-x}}{\Gamma(a+1)} .
$$

The recurrence relations (31) are tested for a large number of random points in the input parameter domain $(a, x), x \leq 0, a>10^{-300}$. The aimed accuracy is close to $10^{-13}$ in most cases. For small values of $a$, the accuracy of the values computed by our routine cdfgamC is even higher, near $10^{-15}$ as can be seen in Table 1. In this table, a comparison of a few values against those obtained using the Matlab function gammainc included in the release $2013 \mathrm{a}$, is shown. It should be noted that versions of gammainc included in some previous Matlab releases showed some loss of accuracy for these same values.

For the inversion algorithm, testing is made by checking that the composition of the functions with their inverse is the identity. Some tests for the inversion routine invedfgamC are shown in Table 2 . This table shows the 


\begin{tabular}{cccccc}
\multicolumn{7}{c}{$a$} \\
\hline$p$ & 0.05 & 1 & 10 & 100 & 1000 \\
\hline 0.0001 & $410^{-16}$ & $4.110^{-16}$ & $6.510^{-15}$ & $8.110^{-16}$ & $5.310^{-15}$ \\
0.01 & $210^{-16}$ & $5.210^{-16}$ & $2.910^{-15}$ & $1.210^{-15}$ & $1.910^{-15}$ \\
0.1 & $2.810^{-16}$ & $1.410^{-16}$ & 0 & $1.510^{-15}$ & $8.310^{-16}$ \\
0.3 & 0 & $1.810^{-16}$ & $3.710^{-16}$ & $7.410^{-16}$ & $7.410^{-16}$ \\
0.5 & $10^{-16}$ & 0 & 0 & $1.110^{-16}$ & $2.210^{-16}$ \\
0.7 & 0 & $1.610^{-16}$ & 0 & $1.610^{-16}$ & $7.910^{-16}$ \\
0.9 & 0 & 0 & 0 & 0 & $3.710^{-16}$ \\
0.9999 & 0 & 0 & 0 & 0 & 0
\end{tabular}

Table 2. Relative errors $\left|P\left(a, x_{r}\right)-p\right| / p$ for several values of $p$ and $a$.

relative error for the inversion problem of the central gamma distribution function given in Eq. (4) for different values of $p$ and $a$. in [6].

Further tests for the central gamma distribution functions can be found

Testing of the noncentral gamma distributions can be made by checking the deviations from 1 of the relation

$$
\frac{(x-\mu) Q_{\mu+1}(x, y)+(y+\mu) Q_{\mu}(x, y)}{x Q_{\mu+2}(x, y)+y Q_{\mu-1}(x, y)}=1 .
$$

Equation (33) is used when $y \geq x+\mu$ and the same expression but for $P_{\mu}(x, y)$ when $y<x+\mu$. The aimed accuracy for the computation of the noncentral gamma cumulative distribution functions is close to $10^{-11}$ in most cases in the parameter domain $(x, y, \mu)$ with $0 \leq x \leq 10000,0 \leq y \leq 10000$ and $0.5 \leq \mu \leq 10000$.

We have compared our routines for the noncentral distributions against the package ncg in $\mathrm{R}$. The description of the algorithms included in this package is given in [19]. Algorithms (but not associated software) for the computation of the noncentral gamma distributions are also described in [20]. The package ncg includes functions for the computation of the noncentral gamma cumulative distribution function (function pgammanc), the computation of the noncentrality parameter (function deltagammanc) and the quantiles of the noncentral gamma distribution function (function qgammanc). In our notation, this corresponds to the computation of the function $P_{\mu}(x, y)$, the inversion of $P_{\mu}(x, y)$ with respect to $x$ and the the inversion of $P_{\mu}(x, y)$ with respect to $y$, respectively. Therefore, there is a first difference between our 


\begin{tabular}{|c|c|c|}
\hline$\mu, x, y$ & $\operatorname{pgammanc}(y, \mu, 2 x)$ & \\
\hline $5,150,30$ & $\mathbf{1 . 1 3 2 4 2 8 6 7 6 1 5 0 \ldots \mathbf { 1 0 } ^ { - \mathbf { 2 3 } }}$ & $\begin{array}{l}\text { GST: } 1.215915354045 \ldots 10^{-23} \\
\text { MATH: } 1.215915354045 . .10^{-23}\end{array}$ \\
\hline $1,75,0.5$ & $1.215915354045 \ldots 10^{-23}$ & $\begin{array}{l}\text { GST: } 3.287840255874 \ldots 10^{-30} \\
\text { MATH: } 3.287840255874 \ldots 10^{-30}\end{array}$ \\
\hline $2,100,2$ & $1.976996751829 \ldots 10^{-38}$ & $\begin{array}{l}\text { GST: } 1.557081489535 \ldots 10^{-35} \\
\text { MATH: } 1.557081489535 \ldots 10^{-35}\end{array}$ \\
\hline $10,100,1$ & $5.486504449527 \ldots 10^{-57}$ & $\begin{array}{l}\text { GST: } 5.152185145235 \ldots 10^{-48} \\
\text { MATH: } 5.152185145235 \ldots 10^{-48}\end{array}$ \\
\hline
\end{tabular}

Table 3. Test of the routine cdfgamNC: comparison of the values obtained for $P_{\mu}(x, y)$ using the $\mathrm{R}$ function pgammanc, our routine cdfgamNC (values GST) and the Mathematica function $N[\operatorname{MarcumQ}[\mu, \sqrt{2 x}, 0, \sqrt{2 y}]$ (values MATH) for some values of $\mu, x$ and $y$.

routines and those included in the package ncg: our algorithms for the direct computation allow to obtain both $P_{\mu}(x, y)$ and its complementary function, the function $Q_{\mu}(x, y)$. Also, the inversion algorithms compute for the minimum of $P_{\mu}(x, y)$ and $Q_{\mu}(x, y)$, which is very convenient for computations in the queues of the distributions.

In the computation of $P_{\mu}(x, y)$, we have checked that the function pgammanc has some problems when computing small values of the cumulative distribution function. This is illustrated in Table 3, where computations by pgammanc, our routine cdfgamNC and the Mathematica function MarcumQ for some values of $\mu, x$ and $y$ are shown.

Regarding the comparison of the inversion routine with respect to $x$ (the noncentrality parameter), the computations using the function deltagammanc included in the package ncg seem to be very slow for certain parameter regions (small $\mu$, large $y$, small $p$ ). For example, for computing $x$ in $P_{1.9}(x, 288)=0.00001$ ( $2 x$ when calling the function deltagammanc) was about $40 \mathrm{~s}$ when using deltagammanc and $10^{-5} \mathrm{~s}$ when using invedfgamNC.

Another test for the inversion of the noncentral gamma distribution func- 


\begin{tabular}{cccc|ccc}
\multicolumn{3}{c|}{ Inv. wrt $x$} & \multicolumn{3}{c}{ Inv. wrt $y$} \\
\hline$q$ & $y=10$ & $y=100$ & $y=1000$ & $x=10$ & $x=100$ & $x=1000$ \\
\hline 0.001 & $410^{-13}$ & $410^{-16}$ & $1.510^{-15}$ & $6.510^{-16}$ & $1.510^{-15}$ & $1.510^{-15}$ \\
0.1 & $210^{-15}$ & $810^{-14}$ & $410^{-14}$ & $2.810^{-16}$ & $8.110^{-14}$ & $2.510^{-13}$ \\
0.3 & $110^{-13}$ & 0 & $310^{-15}$ & $9.310^{-16}$ & $1.510^{-14}$ & $7.310^{-14}$ \\
0.5 & $410^{-15}$ & 0 & 0 & $2.510^{-14}$ & 0 & $210^{-15}$ \\
0.7 & $510^{-15}$ & $810^{-16}$ & $4.910^{-15}$ & $510^{-15}$ & $3.210^{-14}$ & \\
0.999 & $210^{-16}$ & 0 & 0 & 0 & 0 & 0
\end{tabular}

Table 4. Relative errors in the inversion of the noncentral gamma distribution function with respect to (wrt) $x$ and with respect to $y$. The value of $\mu$ has been fixed to 0.5

tion, is shown in Table 4. In this table, the relative error for the inversion problem with respect to $x$ and $y$ of the noncentral gamma distribution function given in Eq. (4) for different values of $(q, x)$ and $(q, y)$ ( $\mu$ fixed to 0.5) is shown. In a second test, $10^{7}$ random points have been generated in the domain of input parameters $\mu \in[0.5,10000], y \in[0,10000](x \in[0,10000])$ and $q \in[0,1]$ for testing the inversion with respect to $x(y)$. Fixing the accuracy parameter of the secant method to $10^{-12}$, the accuracy of the computed values obtained in the inversion was always better than $10^{-11}$.

The accuracy of the routines for the computation of the error function, the complementary error function, the gamma function and its related functions have been tested by comparing the values obtained against Maple (with a large number of digits). In all cases, a full agreement in at least $13-14$ significant digits was found.

Finally, Table 5 shows relative errors in the inversion process of the complementary error function $y=\operatorname{erfc}(x)$ for different values of $y$.

\section{Test run description}

The Fortran 90 test program testgamCHI.f90 includes several tests for the computation of function values and their comparison with the corresponding pre-computed results. Also, the inversion routines are tested by checking that the composition of the function with its inverse is the identity. 


\begin{tabular}{c|c|c|}
$y$ & $\tilde{x}$ & $\|\operatorname{erfc}(\tilde{x}) / y-1\|$ \\
\hline 1.9 & -1.1630871536766738 & 0 \\
1.0 & $-8.57141491386392410^{-17}$ & 0 \\
$10^{-2}$ & 1.163087153676674 & $1.410^{-16}$ \\
$10^{-3}$ & 2.3267537655135246 & $4.310^{-16}$ \\
$10^{-4}$ & 2.7510639057120607 & $2.710^{-16}$ \\
$10^{-5}$ & 3.123413274340875 & $1.210^{-16}$ \\
$10^{-6}$ & 3.4589107372795 & $8.410^{-16}$ \\
$10^{-7}$ & 3.766562581570838 & $6.610^{-15}$ \\
$10^{-8}$ & 4.052237243871389 & $2.110^{-15}$ \\
$10^{-9}$ & 4.320005384913445 & $8.310^{-16}$ \\
$10^{-10}$ & 4.572824967389486 & $4.810^{-15}$ \\
$10^{-11}$ & 4.812924067365823 & $4.810^{-16}$ \\
$10^{-12}$ & 5.042029745639059 & 0
\end{tabular}

Table 5. Solutions $x(y)$ of the equation $y=\operatorname{erfc}(x)$.

\section{Appendix: The asymptotic inversion method}

The cumulative distribution functions considered in this paper can be written in one of the standard forms (see also [21])

$$
\begin{aligned}
F_{a}(\eta) & =\sqrt{\frac{a}{2 \pi}} \int_{-\infty}^{\eta} e^{-\frac{1}{2} a \zeta^{2}} f(\zeta) d \zeta, \\
G_{a}(\eta) & =\sqrt{\frac{a}{2 \pi}} \int_{\eta}^{\infty} e^{-\frac{1}{2} a \zeta^{2}} f(\zeta) d \zeta,
\end{aligned}
$$

where $a>0, \eta \in \mathbb{R}$, and $f$ is analytic and real on $\mathbb{R}$ with $f(0)=1$. The special case $f=1$ gives the normal distributions (see (14))

$$
\begin{aligned}
& P(\eta \sqrt{a})=\sqrt{\frac{a}{2 \pi}} \int_{-\infty}^{\eta} e^{-\frac{1}{2} a \zeta^{2}} d \zeta=\frac{1}{2} \operatorname{erfc}(-\eta \sqrt{a / 2}), \\
& Q(\eta \sqrt{a})=\sqrt{\frac{a}{2 \pi}} \int_{\eta}^{\infty} e^{-\frac{1}{2} a \zeta^{2}} d \zeta=\frac{1}{2} \operatorname{erfc}(+\eta \sqrt{a / 2}) .
\end{aligned}
$$

We explain how the incomplete gamma function $P(a, x)$ defined in (2) can be written in this standard form. Let $\lambda=\frac{x}{a}$ and $t=a \tau$. Then

$$
\Gamma^{*}(a) P(a, x)=\sqrt{\frac{a}{2 \pi}} \int_{0}^{\lambda} e^{-a(\tau-\ln \tau-1)} \frac{d \tau}{\tau},
$$


where $\Gamma^{*}(a)$ is defined in (27). The transformation

$$
\tau-\ln \tau-1=\frac{1}{2} \zeta^{2}, \quad \operatorname{sign}(\tau-1)=\operatorname{sign}(\zeta),
$$

gives the standard form

$$
\Gamma^{*}(a) P(a, x)=\sqrt{\frac{a}{2 \pi}} \int_{-\infty}^{\eta} e^{-\frac{1}{2} a \zeta^{2}} f(\zeta) d \zeta, \quad f(\zeta)=\frac{1}{\tau} \frac{d \tau}{d \zeta}
$$

where $\eta$ is defined by

$$
\lambda-\ln \lambda-1=\frac{1}{2} \eta^{2}, \quad \operatorname{sign}(\lambda-1)=\operatorname{sign}(\eta) .
$$

By using an integration by parts procedure in (34) we can obtain an asymptotic representation of $F_{a}(\eta)$ which is valid for $a \rightarrow \infty$, uniformly for all $\eta \in \mathbb{R}$. We write $f(\eta)=[f(\eta)-f(0)]+f(0)$, where $f(0)=1$, and use (35). Then we obtain by repeating integration by parts

$$
\begin{aligned}
& F_{a}(\eta)=\frac{1}{2} \operatorname{erfc}(-\eta \sqrt{a / 2}) F_{a}(\infty)+\frac{e^{-\frac{1}{2} a \eta^{2}}}{\sqrt{2 \pi a}} S_{a}(\eta), \\
& G_{a}(\eta)=\frac{1}{2} \operatorname{erfc}(\eta \sqrt{a / 2}) G_{a}(-\infty)-\frac{e^{-\frac{1}{2} a \eta^{2}}}{\sqrt{2 \pi a}} S_{a}(\eta),
\end{aligned}
$$

where $F_{a}(\infty)=G_{a}(-\infty)$ and

$$
F_{a}(\infty) \sim \sum_{n=0}^{\infty} \frac{A_{n}}{a^{n}}, \quad A_{0}=1, \quad S_{a}(\eta) \sim \sum_{n=0}^{\infty} \frac{C_{n}(\eta)}{a^{n}}, \quad a \rightarrow \infty,
$$

uniformly with respect to $\eta \in \mathbb{R}$. The coefficients follow from the following recursive scheme. Let $f_{0}(\eta)=f(\eta)$. Then, for $n=0,1,2, \ldots$, define

$$
f_{n+1}(\eta)=\frac{d}{d \eta} \frac{f_{n}(\eta)-f_{n}(0)}{\eta}
$$

and we have

$$
A_{n}=f_{n}(0), \quad C_{n}(\eta)=\frac{f_{n}(0)-f_{n}(\eta)}{\eta} .
$$

To describe the inversion problem, we assume that $p \in(0,1)$ and that $a$ is a large positive parameter. Then we are interested in the value $\eta$ that solves the equation

$$
F_{a}(\eta)=F_{a}(\infty) p
$$


where $F_{a}(\eta)$ has the form given in (40).

First we define a number $\eta_{0}$ that solves the reduced equation

$$
\frac{1}{2} \operatorname{erfc}\left(-\eta_{0} \sqrt{a / 2}\right)=p .
$$

Then for the desired value $\eta$ we assume the expansion

$$
\eta \sim \eta_{0}+\frac{\eta_{1}}{a}+\frac{\eta_{2}}{a^{2}}+\frac{\eta_{3}}{a^{3}}+\ldots, \quad a \rightarrow \infty,
$$

and try to find the coefficients $\eta_{1}, \eta_{2}, \eta_{3}, \ldots$. To obtain the $\eta_{j}$ we can substitute the expansion for $\eta$ into the asymptotic expansion of $F_{a}(\eta)$ and use formal power series manipulations.

However, here we use the method as explained in our earlier mentioned publications. This method runs as follows. From (34), (44), and (45) we obtain

$$
\frac{d p}{d \eta_{0}}=\sqrt{\frac{a}{2 \pi}} e^{-\frac{1}{2} a \eta_{0}^{2}}, \quad \frac{d p}{d \eta}=\sqrt{\frac{a}{2 \pi}} \frac{f(\eta)}{F_{a}(\infty)} e^{-\frac{1}{2} a \eta^{2}},
$$

from which we obtain, upon dividing,

$$
f(\eta) \frac{d \eta}{d \eta_{0}}=F_{a}(\infty) e^{\frac{1}{2} a\left(\eta^{2}-\eta_{0}^{2}\right)} .
$$

Substituting (46) and using $F_{a}(\infty)=1+\mathcal{O}(1 / a)$, we obtain, after first-order perturbation analysis for large $a$,

$$
f\left(\eta_{0}\right)=e^{\eta_{0} \eta_{1}} \quad \Longrightarrow \quad \eta_{1}=\frac{1}{\eta_{0}} \ln f\left(\eta_{0}\right) .
$$

Because $f$ is analytic at the origin with $f(0)=1, \eta_{1}$ is well defined as $\eta_{0} \rightarrow 0$.

For higher-order terms $\eta_{j}, j \geq 2$, we need in (48) more coefficients in the asymptotic expansion of $F_{a}(\infty)$ (see (41)) and we have to expand

$$
f(\eta)=f\left(\eta_{0}\right)+\left(\eta-\eta_{0}\right) f^{\prime}\left(\eta_{0}\right)+\frac{1}{2}\left(\eta-\eta_{0}\right)^{2} f^{\prime \prime}\left(\eta_{0}\right)+\ldots
$$

Then, the next coefficients are given by

$$
\begin{aligned}
\eta_{2}= & -\left(f\left(2 A_{1}+\eta_{1}^{2}-2 \eta_{1}^{\prime}\right)-2 \eta_{1} f^{\prime}\right) /\left(2 \eta_{0} f\right), \\
\eta_{3}= & -\left(8 f \eta_{1} \eta_{2}+4 A_{1} f \eta_{1}^{2}+f \eta_{1}^{4}+4 f \eta_{1}^{2} \eta_{0} \eta_{2}+4 f \eta_{0}^{2} \eta_{2}^{2}-8 f \eta_{2}^{\prime}+\right. \\
& \left.8 A_{1} f \eta_{0} \eta_{2}+8 A_{2} f-8 f^{\prime} \eta_{1} \eta_{1}^{\prime}-8 f^{\prime} \eta_{2}-4 f^{\prime \prime} \eta_{1}^{2}\right) /\left(8 \eta_{0} f\right),
\end{aligned}
$$


where $f, f^{\prime}$ and $f^{\prime \prime}$ are evaluated at $\eta_{0}$.

For small values of $\eta_{0}$ (that is, when $p \sim \frac{1}{2}$ ), we need expansions. We have

$$
\begin{aligned}
& \eta_{1}=a_{1}+\frac{1}{2}\left(2 a_{2}-a_{1}^{2}\right) \eta_{0}+\frac{1}{3}\left(3 a_{3}-3 a_{1} a_{2}+2 a_{1}^{3}\right) \eta_{0}^{2}+\mathcal{O}\left(\eta_{0}^{3}\right), \\
& \eta_{2}=-\frac{1}{3} a_{1}^{3}+2 a_{3}+\frac{1}{8}\left(-12 a_{2} a_{1}^{2}+5 a_{1}^{4}+24 a_{4}\right) \eta_{0}+\mathcal{O}\left(\eta_{0}^{2}\right), \\
& \eta_{3}=\frac{1}{15}\left(4 a_{1}^{5}-5 a_{2} a_{1}^{3}-15 a_{3} a_{1}^{2}+120 a_{5}\right)+\mathcal{O}\left(\eta_{0}\right),
\end{aligned}
$$

where $a_{k}$ are the coefficients in the expansion $f(\zeta)=\sum_{k=0}^{\infty} a_{k} \zeta^{k}$. The $a_{k}$ are related to the $A_{k}$ used in (51):

$$
A_{n}=\left(\frac{1}{2}\right)_{n} 2^{n} a_{2 n}, \quad n=0,1,2,, \ldots
$$

\section{Acknowledgements}

The authors acknowledge financial support from Ministerio de Ciencia e Innovación, project MTM2012-11686. NMT thanks CWI, Amsterdam, for scientific support.

\section{References}

[1] A. Gil, J. Segura, N. M. Temme, The asymptotic and numerical inversion of the Marcum Q-function, Studies in Applied Mathematics 133 (2014) $257-278$.

[2] X. Tang, Y. Peida, Calculation of the bit-error rate for optical soliton communication systems with lumped amplifiers, Optics Letters 18 (1993) 1156-1158.

[3] R. F. Mills, G. E. Prescott, A comparison of various radiometer detection models, IEEE Trans. Aerosp. Electron. Syst. 32 (1996) 467-473.

[4] C. W. Helstrom, Quantum detection and estimation theory, Academic Press, 1976.

[5] D. Y. Vasylyev, A. A. Semenov, W. Vogel, Toward Global Quantum Communication: Beam Wandering Preserves Nonclassicality, Phys. Rev. Lett. 108 (2012) 220501. 
[6] A. Gil, N. M. Temme, J. Segura, Efficient and accurate algorithms for the computation and inversion of the incomplete gamma function ratios, SIAM J. Sci. Comput. 34 (6) (2012) A2965-A2981.

[7] J. Segura, Monotonicity properties and bounds for the chi-square and gamma distributions, Appl. Math. Comput. 246 (2014) 399-415.

[8] A. Gil, J. Segura, N. M. Temme, Algorithm 939: Computation of the Marcum Q-function, ACM Trans Math Soft 40 (3) (2014) Article 20.

[9] J. Segura, The Schwarzian-Newton method for solving nonlinear equations, with applications (in preparation).

[10] N. M. Temme, Error functions, Dawson's and Fresnel integrals, in: NIST handbook of mathematical functions, U.S. Dept. Commerce, Washington, DC, 2010, pp. 159-171.

[11] W. Cody, Rational Chebyshev approximations for the error function, Math Comput 23 (1969) 631-637.

[12] A. Gil, J. Segura, N. M. Temme, Numerical methods for special functions, SIAM, Philadelphia, PA, 2007.

[13] A. Strecok, On the calculation of the inverse of the error function, Math. Comp. 22 (1968) 144-158.

[14] H. Hochchstadt, The functions of mathematical physics, John Wiley, 1971.

[15] F. Olver, D. Lozier, R. Boisvert, C. Clark, (Eds). NIST handbook of mathematical functions, U.S. Dept. Commerce, Washington, DC, 2010.

[16] J. F. Hart, E. W. Cheney, C. L. Lawson, H. J. Maehly, C. K. Mesztenyi, J. R. Rice, H. G. Thacher, Jr., C. Witzgall, Computer approximations., The SIAM Series in Applied Mathematics. New York etc: John Wiley \& Sons, Inc. X, 343 p. (1968). (1968).

[17] N. M. Temme, Special functions: An introduction to the classical functions of mathematical physics, A Wiley-Interscience Publication, John Wiley \& Sons Inc., New York, 1996. 
[18] R. B. Paris, Incomplete gamma and related functions, in: NIST handbook of mathematical functions, U.S. Dept. Commerce, Washington, DC, 2010, pp. 175-192.

[19] I. Oliveira, D. F. Ferreira, Computing the noncentral gamma distribution, its inverse and the noncentrality parameter, Comput Stat 28 (2013) 1663-1680.

[20] L. Knüsel, B. B., Computation of the noncentral gamma distribution, SIAM J Sci Comput 17 (5) (1996) 1224-1231.

[21] A. Gil, J. Segura, N. M. Temme, The asymptotic inversion of certain cumulative distribution functions, in: A. D. Fitt, J. Norbury, H. Ockendon, E. Wilson (Eds.), Progress in Industrial Mathematics at ECMI 2008, Springer-Verlag, 2010, pp. 117 -122 .

URL http://oai.cwi.nl/oai/asset/16430/16430A.pdf 